\title{
Water diffusion in rat brain in vivo as detected at very large $b$ values is multicompartmental
}

\author{
Josef Pfeuffer ${ }^{\mathrm{a}, *}$, Stephen W. Provencher ${ }^{\mathrm{b}}$, Rolf Gruetter ${ }^{\mathrm{a}}$ \\ a Department of Radiology, University of Minnesota Medical School, Center for Magnetic Resonance Research, 2021 Sixth Street S.E., \\ Minneapolis, MN 55455, USA \\ b Max Planck Institute for Biophysical Chemistry, Postfach 2841, D-37070 Göttingen, Germany
}

Received 1 October 1998; received in revised form 28 January 1999; accepted 18 February 1999

\begin{abstract}
The diffusion-weighted signal attenuation of water in rat brain was measured with pulsed-field gradient nuclear magnetic resonance methods in a single voxel under in vivo and global ischemic conditions. The diffusion-attenuated water signal was observed in vivo at $b$ values of $300 \mathrm{~ms} / \mu \mathrm{m}^{2}$ (strength of diffusion weighting) and diffusion times up to $400 \mathrm{~ms}$. A series of constant diffusion time (CT) experiments with varied gradient directions and diffusion times revealed a multiexponential decay with

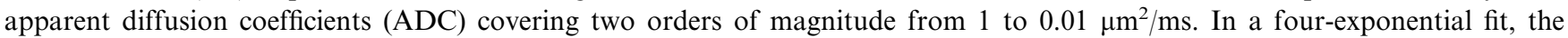
observed changes during global ischemia could be fully explained by changes in the relative volume fractions only with unchanged ADCs. An anisotropy of the ADC, detected at small $b$ values, was not observed for the ADC at large $b$ values, but for the concomitant volume fractions. An inverse Laplace Transform of the CT curves, performed with CONTIN, resulted in continuously distributed diffusion coefficients, for which the term 'diffusogram' is proposed. This approach was more appropriate than a discrete exponential model with four to six components, being related to the morphology of brain tissue and its cell size distribution. On the basis of an analytical, quantitative model, it is suggested that the measured ADC at small $b$ values reflects mainly properties of the restricting boundaries, i.e. the relative volume fractions and the extracellular tortuosity, while the intrinsic intracellular diffusion constant and the exchange time are predicted to have minor influence. C) 1999 Elsevier Science B.V. All rights reserved.
\end{abstract}

Keywords: Multiexponential decay; Restricted diffusion; Diffusion time dependence; Anisotropy; Rat brain; Diffusogram

\section{Introduction}

Pulsed-field-gradient nuclear magnetic resonance (NMR) methods are widely applicable to assess the diffusion of water and metabolites in biological systems $[1,2]$. For clinical diagnosis, especially, diffusionweighted imaging is used to generate a contrast due to differences in the apparent diffusion coefficient (ADC, $D^{\text {app}}$ ), which is sensitive to detecting some brain pathologies, e.g. brain lesions or cytotoxic edema in a very early stage of stroke [3]. Changes of the ADC during cerebral ischemia are affected by many compartmental properties of brain tissue, i.e. pathological changes of volume fraction and morphology [4], extra-

\footnotetext{
* Corresponding author. Tel.: + 1-612-625-7897; fax: + 1-612-6262004.

E-mail address: pfeuffer@cmrr.umn.edu (J. Pfeuffer)
}

cellular tortuosity [5-7], intracellular restriction [8,9], transmembrane exchange [10-12], relaxation [13], and active transport processes [14], etc. These multiple effects complicate the interpretation of diffusion data and require sophisticated experiments and tissue models to separate their influence on the water diffusion attenuation.

Many different experiments were reported to approach the understanding of the diffusion-weighted contrast in tissue, studies in cell cultures, excised tissue and nerves, animals, and humans. Analytical models and simulations involved multiple compartments, exchange, directional anisotropy, and $T_{2}$ relaxation effects [11,15-19]. Important experimental features were gained by measurements depending on the gradient direction (anisotropy of the diffusion tensor) and on the diffusion time (from 5 to $2000 \mathrm{~ms}$ ), and extending the 
strength of the diffusion weighting to the largest possible $b$ values [20-28]. It was shown that the diffusion attenuation in vivo is no longer monoexponential at larger $b$ values, which is connected with structural information and diffusion dynamics in analogy to porous media [8,29-33].

Previous experimental and theoretical work on diffusion modeling was done with perfused glial cells and in rat brain $[12,18,34-36]$. The concept of 'restricted intracellular water diffusion at permeable boundaries' was able to explain diffusion time dependent data of cell cultures, including the transition from intracellular free to restricted diffusion as well as the water exchange across the membrane [18]. The model and parameters were adapted to the situation in rat brain tissue and combined with the concept of 'extracellular tortuosity'. It then described quantitatively the non-monoexponentiality and the diffusion time dependence of the signal attenuation curves, as well as the absolute value of the $\mathrm{ADC}$ at small $b$ values and its decrease during ischemia by changes of the intracellular volume fraction, the extracellular tortuosity, and the intracellular exchange time [12].

The purpose of the present study was to extend the experimental possibilities and to perform localized diffusion-weighted ${ }^{1} \mathrm{H}$ single voxel experiments in vivo at very large $b$ values, at different directions, and at large diffusion times. A multicompartmental behavior of the diffusion attenuation was characterized by a continuous distribution of diffusion coefficients, for which the term 'diffusogram' is proposed. A tissue model was applied to determine the influences on the signal attenuation curves and the apparent diffusion coefficient at small $b$ values. The structural information of the restricting

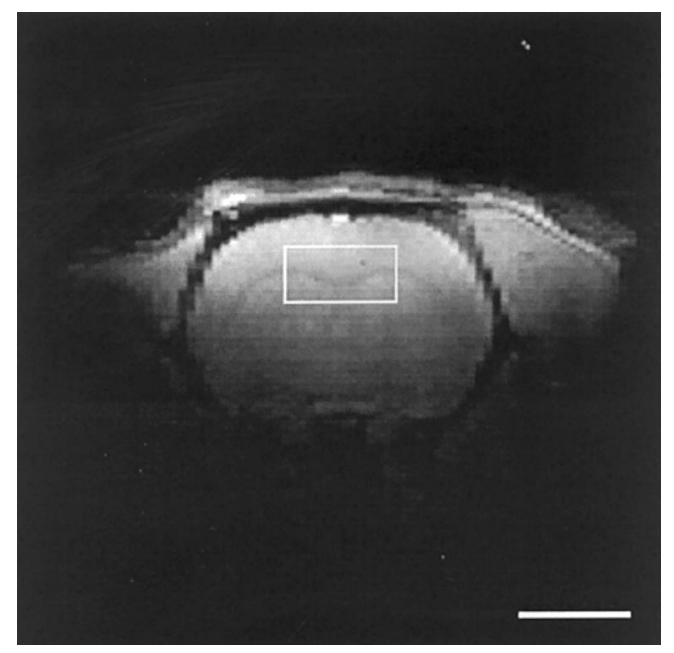

Fig. 1. FLASH image of the rat brain in vivo indicating the voxel-ofinterest with size $5 \times 2.5 \times 5 \mathrm{~mm}^{3}$ (field-of-view $3 \mathrm{~cm}$, scale bar 5 $\mathrm{mm}$ ). The voxel was positioned on the midline $2 \mathrm{~mm}$ posterior to bregma and $3 \mathrm{~mm}$ ventral, and included tissue from cortex, hippocampus, and striatum. boundaries had to be included therein, i.e. the cell walls and the volume fraction of the accompanying compartments, and its changes during pathological tissue states.

\section{Methods}

Male Sprague-Dawley rats (230-280 g, $n>6)$ were anesthetized with $2 \%$ Isoflurane in $60: 40 \% \mathrm{O}_{2}: \mathrm{N}_{2} \mathrm{O}$, and ventilated at physiological conditions. The oxygen saturation, maintained above $95 \%$, was continuously monitored with an IR sensor clipped to the tail (Nonin Medical, Minneapolis, MN). A heated water circuit was controlled by a rectal thermosensor (Cole-Parmer, Vernon Hills, IL) to stabilize the body temperature at $37^{\circ} \mathrm{C}$. A femoral venous line was used to infuse highdosed potassium chloride for euthanasia, which instantaneously induced cardiac arrest followed by a global cerebral ischemia. The blood pressure and heart beat were continuously monitored by a blood pressure transducer connected to a femoral arterial line.

The experiments were performed with a Varian INOVA spectrometer (Varian, Palo Alto, CA) interfaced to a 31-cm horizontal bore, 9.4 Tesla magnet, which was equipped with an $11-\mathrm{cm}$ actively-shielded gradient insert capable of switching $300 \mathrm{mT} / \mathrm{m}$ in $500 \mu \mathrm{s}$ in $x, y$, and $z$ direction (Magnex Scientific, Abingdon, UK). The quadrature $400 \mathrm{MHz}{ }^{1} \mathrm{H}$ surface coil for r.f. transmit and receive as well as further experimental details were described previously [37]. The localization was performed by a STEAM [38] sequence using a $5 \times 2.5 \times 5 \mathrm{~mm}^{3}$ voxel (position shown in Fig. 1). After careful eddy current compensation [39], shimming of the first- and second-order shim coils was done with FASTMAP [40], typically resulting in a $12-13 \mathrm{~Hz}$ linewidth of the water resonance.

\subsection{Diffusion weighting}

To minimize relaxation losses due to $T_{2}$, unipolar gradients for diffusion weighting of the ${ }^{1} \mathrm{H}$ water signal were placed during $\tau$ in the stimulated echo sequence $90^{\circ}-\tau-90^{\circ}-\mathrm{TM}-90^{\circ}-\tau$ with middle time $\mathrm{TM}$ and echo time $\mathrm{TE}=2 \tau$. The experimental parameters determining the ${ }^{1} \mathrm{H}$ water diffusion attenuation were the gradient duration $\delta$, the gradient strength $G$, and the separation $\Delta$ of the leading edges of the gradients. The $q$ value was defined as $q=\left(\gamma^{2} G^{2} \delta^{2}\right)$, the diffusion time as $t_{\mathrm{D}}=\Delta-$ $\delta / 3$, and the $b$ value as $b=q \cdot t_{\mathrm{D}}$. For Gaussian diffusion, the relation between signal attenuation and diffusion coefficient $\ln S / S_{0}=-b \cdot D$ holds. While keeping TE constant, $T_{2}$ relaxation effects were excluded, but variation of the diffusion time and thereby TM involved an additional attenuation due to $T_{1}$ relaxation. 


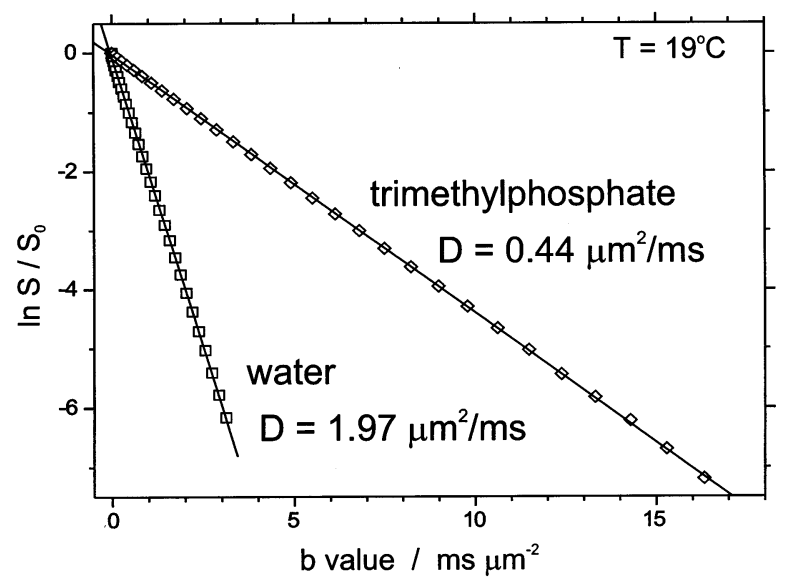

Fig. 2. Diffusion-weighted ${ }^{1} \mathrm{H}$ signal attenuation of water and trimethylphosphate in phantoms at $19^{\circ} \mathrm{C}$ from a $63 \mu \mathrm{l}$ voxel. The monoexponentiality was observed up to an attenuation of $\exp (-7.1)$, which corresponded to a signal-to-noise ratio of more than 1300 (diffusion time $t_{\mathrm{D}}=28$ and $26 \mathrm{~ms}$, gradient length $\delta=5$ and $13 \mathrm{~ms}$, echo time $\mathrm{TE}=20$ and $35 \mathrm{~ms}$, number of scans $\mathrm{nt}=1$, repetition time $\mathrm{TR}=4 \mathrm{~s})$.

In a constant diffusion time (CT) experiment, $G$ was varied in different directions by 32 or 128 linearly spaced steps, $t_{\mathrm{D}}$ and $\delta$ were kept constant. In a constant gradient (CG) experiment, $t_{\mathrm{D}}$ was varied and $G$ and $\delta$ were kept constant. For single signal monitoring, the diffusion weighting was kept constant with fixed $G, \delta$, and $t_{\mathrm{D}}$, and the signal was repeatedly measured. All scans were recorded separately, which allowed phase tracking prior to signal averaging. After automatic phase correction of the Fourier transformed data, the peak area of the ${ }^{1} \mathrm{H}$ water resonance was determined by a five-point Newton-Cotes integration formula. The software for processing and data conversion was written with PV-WAVE (Visual Numerics, Boulder, CO).

\subsection{Data analysis}

An apparent diffusion coefficient, $D^{\text {app }}$, was calculated from the negative slope of the signal decay vs. $b$ value by log-linear regression. The common term ADC was used for the apparent diffusion coefficient when the fit range was at small diffusion weighting, i.e. $b \rightarrow 0$. The apparent displacement was calculated from CT experiments to $r^{\text {app }}=\left(6 \cdot D^{\text {app }} \cdot t_{\mathrm{D}}\right)^{1 / 2}$, the mean intracellular residence time $\tau^{\text {app }}$ was calculated from the negative reciprocal slope of $\mathrm{CG}$ experiments vs. diffusion time at large $q$ values (see Ref. [18] for details).

An automatic analysis of the discrete components of the multicomponent exponential decay data (i.e. the diffusion-weighted signal attenuation $S$ vs. $b$ value) was performed by DISCRETE [41,42], which determined the number of components, the decay rates (i.e. $D^{\text {app}}$ ), and its relative fractions $p^{\text {app }}$ with $S / S_{0}=\Sigma p_{i}^{\text {app }} \exp (-$ $\left.b \cdot D_{i}^{\text {app }}\right), \Sigma p_{i}^{\text {app }}=1$. Also, the probability was calculated that a solution with certain number of components is better than the second best solution, etc., as well as the statistical errors of all extracted parameters. A simultaneous analysis of a pair of diffusion attenuation curves (healthy and global ischemic rat) with the same exponential decay rates was performed by SplMod [43]. As with DISCRETE, the number of components, the decay rates $D^{\text {app }}$, and the relative fractions $p^{\text {app }}$ of both curves were estimated from a multiexponential non-linear least-squares analysis.

To assess a quasi-continuous distribution of diffusion coefficients in the signal attenuation of a CT experiment, an inverse Laplace Transform was performed with CONTIN $[44,45]$, which determined the relative fractions of the exponential components as well as the number of main peaks, their moments, their mean, their fractions, and their coefficient of variation. Inverse Laplace transforms can describe very complex (even non-exponential) processes and are often useful as a phenomenological description. The results are presented in a histogram plot of the relative fractions of $D^{\text {app }}$ in the signal attenuation curve and in a table summarizing the most relevant quantities. In analogy to the analysis of continuously distributed relaxation parameters (relaxogram) [46], we termed the plot of the distributed diffusion coefficients from the CONTIN analysis of diffusion attenuation data 'diffusogram'. Data points for $b<0.36 \mathrm{~ms} / \mu \mathrm{m}^{2}$ carrying fast decaying components, which were due to experimental imperfections and which were also seen in the phantom data, were omitted from the analysis. The program packages of DISCRETE, SplMod and CONTIN are available at http:// www.provencher.de.

\section{Results}

Verifying the experimental set-up, the signal attenuation of the water and trimethylphosphate phantoms was strictly monoexponential with diffusion constants of $D=1.97 \pm 0.006$ and $0.437 \pm 0.0006 \mu \mathrm{m}^{2} / \mathrm{ms}$ at $19^{\circ} \mathrm{C}$ (Fig. 2). The maximum attenuation of $\exp (-7.1)$ of the signal at $b=0$ corresponded to a signal-to-noise ratio of more than 1300 for one scan. A slight deviation from monoexponentiality was observed for $b \rightarrow 0$.

Fig. 3 shows that the ${ }^{1} \mathrm{H}$ water signal was detected in rat brain in vivo at very large diffusion weighting $(b$ value of $300 \mathrm{~ms} / \mu^{2}$ ) with a reasonable signal-to-noise ratio using eight accumulations. In this CG experiment, the diffusion time $t_{\mathrm{D}}$ was varied from 55 to $395 \mathrm{~ms}$. An apparent diffusion coefficient, $D_{\mathrm{CG}}^{\mathrm{app}}$ of $0.010 \pm 0.001$ $\mu \mathrm{m}^{2} / \mathrm{ms}$ was calculated, which is a factor of 300 lower than the free self-diffusion constant of water at $37^{\circ} \mathrm{C}$ [47]. The signals in Fig. 3, which were measured at a fixed $q$ value of $0.742 \mu \mathrm{m}^{-2}$ and which decayed with increasing diffusion time, were also used to determine 
the apparent mean intracellular residence time $\tau^{\text {app }}$ to be $135 \pm 12 \mathrm{~ms}$.

\subsection{Multicompartmentation in tissue}

The multiexponentiality of the water diffusion attenuation curves in rat brain was assessed by $\mathrm{CT}$ experiments with $b$ values up to $70 \mathrm{~ms} / \mu \mathrm{m}^{2}$ (Fig. 4). The gradient strength was increased independently in $x, y$, and $z$ directions in 128 steps while keeping $\delta, t_{\mathrm{D}}$, and TE constant. In the healthy rat brain, CT curves were recorded subsequently with four accumulations. The data from the global ischemic rat brain were acquired shortly after cardiac arrest with two accumulations (total time $51 \mathrm{~min}$ ), whereby the body temperature,

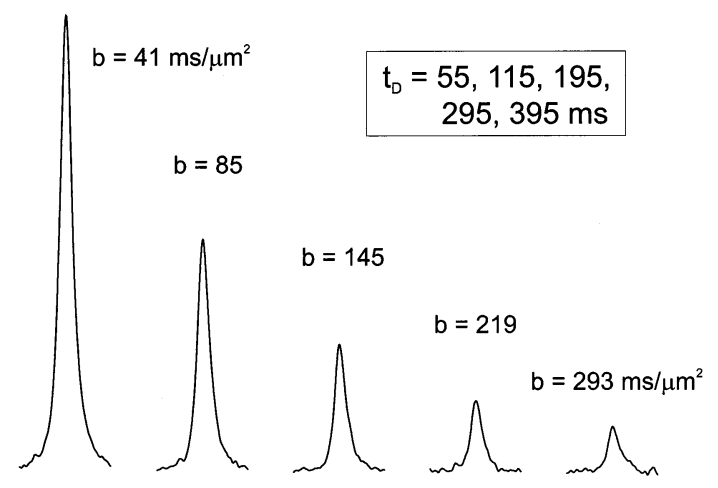

Fig. 3. Diffusion-weighted ${ }^{1} \mathrm{H}$ resonances of water in rat brain in vivo localized by STEAM in a $63 \mu \mathrm{l}$ voxel as measured at very large $b$ values. The experiment was performed with constant gradients (CG) at a fixed $q$ value of $0.742 \mu \mathrm{m}^{-2}$ and varied diffusion times $t_{\mathrm{D}}=55$, $115,195,295$, and $395 \mathrm{~ms}$ (gradient strength $G_{x}=230 \mathrm{mT} / \mathrm{m}, \delta=14$ $\mathrm{ms}, \mathrm{TE}=35 \mathrm{~ms}, \mathrm{nt}=8, \mathrm{TR}=4 \mathrm{~s}$ ).

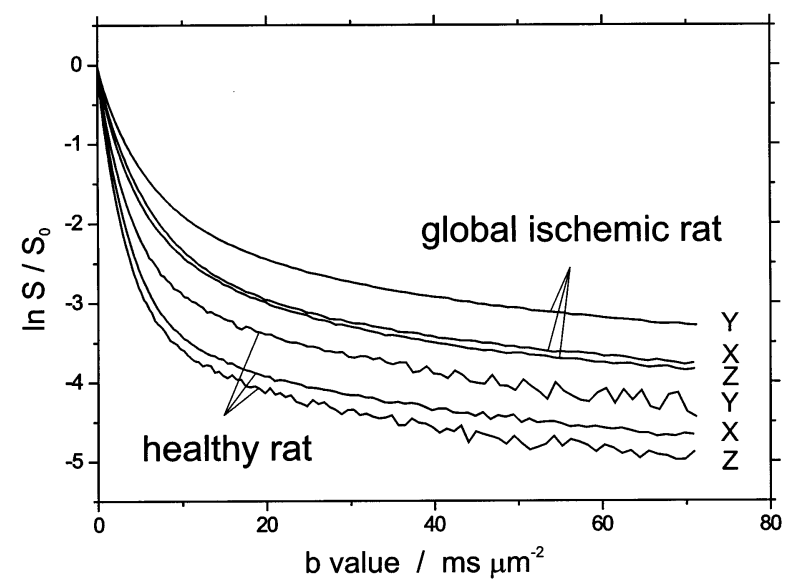

Fig. 4. Water signal attenuation in rat brain in vivo and ex vivo with $b$ values up to $70 \mathrm{~ms} / \mu^{2}$ from a single $63 \mu \mathrm{l}$ voxel. Constant diffusion time (CT) experiments with 128 gradient steps each were performed in $x, y$, and $z$ directions of the healthy rat brain $(\mathrm{nt}=4)$ and of the global ischemic rat brain within $1 \mathrm{~h}$ after cardiac arrest $(\mathrm{nt}=2)$. The data points of the CT curves were normalized by $S(b=0)$ and were linearly connected to show the tiny differences at small $b$ values $\left(\delta=14 \mathrm{~ms}, t_{\mathrm{D}}=115 \mathrm{~ms}, \mathrm{TE}=35 \mathrm{~ms}, \mathrm{TR}=4 \mathrm{~s}\right)$. measured by a rectal sensor, was kept constant within $0.5^{\circ} \mathrm{C}$ to prevent a rapid cooling of the brain during ischemia.

An automatic analysis as a multicomponent exponential decay using DISCRETE yielded four significant components characterized by $D^{\text {app }}$ and its relative fraction $p^{\text {app }}$ (not shown). The two main components had diffusion coefficients $D_{1}^{\text {app }}$ and $D_{2}^{\text {app }}$ of $0.3-1.1 \mu \mathrm{m}^{2} / \mathrm{ms}$ with a total fraction of about $80-95 \%$. Two slowly decaying components, $D_{3}^{\text {app }}$ and $D_{4}^{\text {app }}$, were found in the order of 0.1 and $0.01 \mu \mathrm{m}^{2} / \mathrm{ms}$. Comparing healthy and global ischemic rat brain, $D_{1}^{\text {app }}$ and $D_{2}^{\text {app }}$ were not significantly decreased during ischemia. However, the volume fractions $p_{1}^{\text {app }}$ and $p_{2}^{\text {app }}$ significantly decreased and increased, respectively. Whereas $D_{3}^{\text {app }}$ and $D_{4}^{\text {app }}$ also did not vary significantly in healthy and ischemic brain, the volume fractions $p_{3}^{\text {app }}$ and $p_{4}^{\text {app }}$ increased by about a factor of three in the global ischemic brain.

To compare with the discrete analysis, a continuous distribution of diffusion coefficients was calculated by an inverse Laplace transform from the CT curves of the healthy and global ischemic rat brain in the $x$ direction (Fig. 5A). In addition, the number of peaks in the distribution of $D^{\text {app }}$, their mean, and their relative fractions were determined by the general purpose constrained regularization program CONTIN as shown in Table 1. In the diffusogram of both the healthy and global ischemic rat brain data, a broad distribution of $D^{\text {app }}$ with three maxima could be observed. To illustrate the resolution attainable, the two peaks obtained from the monoexponential phantom data of are shown as dotted curves in Fig. 5A. The most dominant fraction of diffusion coefficients $D_{1}^{\text {app }}$ (about 93\%) was located in the range $0.5-2.0 \mu \mathrm{m}^{2} / \mathrm{ms}$ (healthy rat) and in the range $0.3-1.2 \mu \mathrm{m}^{2} / \mathrm{ms}$ (global ischemic rat) as determined from the coefficient of variation $\sigma$, which is about the peak width at half maximum.

Considering also the statistical output from CONTIN, the following hints were gained from the diffusograms: (1) the mean of the major peak with $D^{\text {app }}$ approximately $1 \mu \mathrm{m}^{2} / \mathrm{ms}$ decreased during global ischemia; (2) no significant change in $D^{\text {app }}$ was observed for the two other peaks with $D^{\text {app }}$ approximately 0.1 and 0.01 $\mu \mathrm{m}^{2} / \mathrm{ms}$; (3) the volume fraction of the latter peaks increased nearly a factor two during global ischemia; (4) a diffusogram as a quasi-continuous distribution was more appropriate for exponential analysis than a fit to discrete components. In the CONTIN reference solution (with best fit to the data), which gave the global optimum for a fit of a discrete sum of exponentials, at least six components were found for the tissue data. Therefore, it seemed more reasonable to use the simpler model of three peaks with effectively continuous distributions.

As seen from the DISCRETE analysis and from peaks (2) and (3) of the CONTIN analysis in Table 1, the 

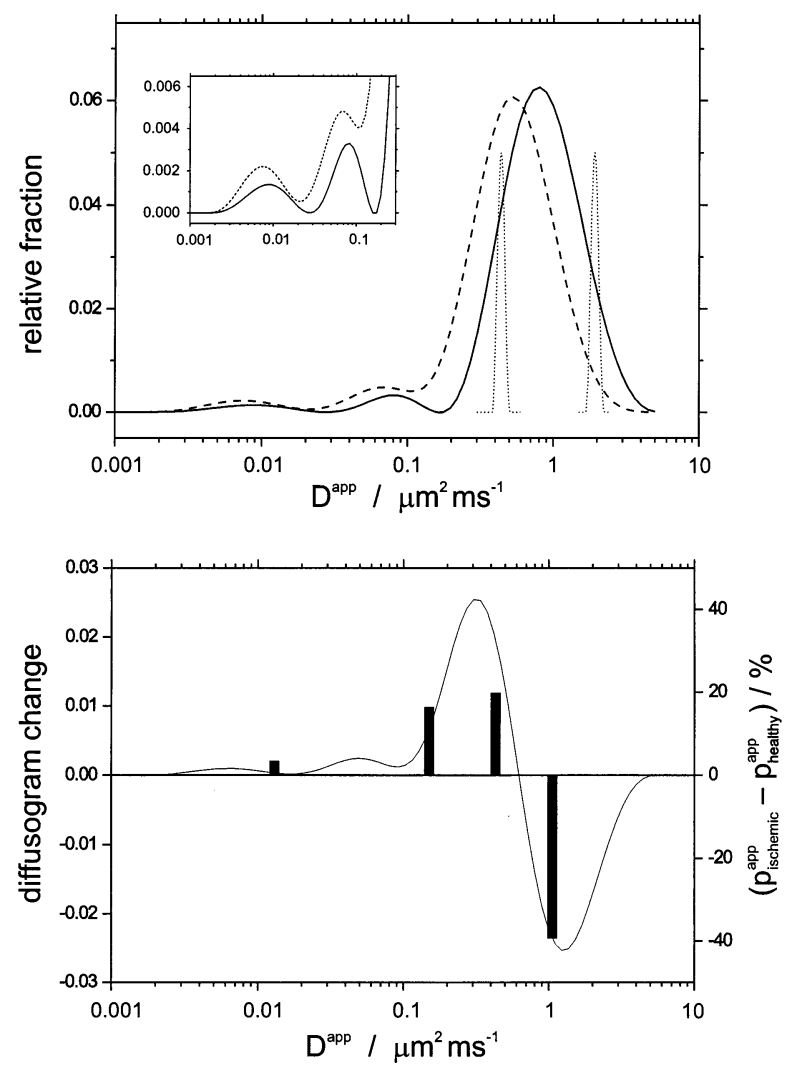

Fig. 5. (A) Diffusogram, i.e. a histogram plot of the relative fractions of quasi-continuously distributed diffusion coefficients $D^{\text {app }}$ in healthy (solid curve) and global ischemic rat brain (dashed curve), calculated by an inverse Laplace transform from the CT curves in the $x$ direction (Fig. 4). Clearly, a broad distribution of $D^{\text {app }}$ with three peaks could be recognized due to the influence of morphology and size distribution on the restricted diffusion behavior in tissue. The two sharp peaks (dotted curve) demonstrate the resolution with an analysis of monoexponential phantom data of water and trimethylphosphate of Fig. 1. (B) The difference diffusogram of the ischemic and healthy rat is shown together with bars, which represent the four discrete components with the simultaneous fitted $D^{\text {app }}$ and the difference between the volume fractions $p^{\text {app }}$ of the ischemic and healthy rat (Table 2). The SplMod and CONTIN analysis are consistent when the changes during ischemia are interpreted predominantly by changes in the volume fractions $p^{\text {app }}$ rather than by a decrease of the apparent diffusion coefficients. Numerical quantities are given in Table 1 . diffusion coefficients did not change significantly during ischemia, which supports the hypothesis that a change in volume fractions may be sufficient to explain the changes in the diffusion attenuation curves. To further test this hypothesis, pairs of healthy and global ischemic data were analyzed simultaneously with SplMod. The optimized parameters were again $D^{\text {app }}$ and $p^{\text {app }}$, except that a strong additional restriction was imposed: $D^{\text {app }}$ was the same in the healthy and ischemic state, with only $p^{\text {app }}$ changing, as might be expected if ischemia produced only shifts in compartmental volumes. The fitted results for all directions are shown in Table 2. The residuals of the fit and the error estimates of the fitted parameters in Table 2 indicate that this model, without changes in $D^{\text {app }}$, can explain the experimental results very well.

To compare the results of the SplMod and CONTIN analysis, the changes of the quantities from the healthy to the global ischemic situation in the $x$ direction are plotted in Fig. 5B. The solid curve in Fig. 5B is the difference between the diffusograms of the ischemic and healthy rat in Fig. 5A. The bars represent the four discrete components with the simultaneously fitted $D^{\text {app }}$ and the difference in the volume fractions $p^{\text {app }}$ of the ischemic and healthy rat (Table 2). In this representation, the decrease in intensity of component (1) at 1 $\mu \mathrm{m}^{2} / \mathrm{ms}$ and the increase in intensity of components (2), (3), and (4) are very consistent in the DISCRETE, SplMod, and CONTIN analysis.

\subsection{Dynamic monitoring during ischemia}

Additional information was expected from a time course of the changes of the highly diffusion-weighted signal during global ischemia, which might be useful for the diffusion modeling when combined with physiological mechanisms. The increase of the strongly diffusionweighted water signal from healthy to the global ischemic rat brain was directly determined by time-resolved single signal monitoring (Fig. 6). Measurements were performed every $2 \mathrm{~s}$ at fixed $b=13.3 \mathrm{~ms} / \mu \mathrm{m}^{2}$ without averaging. The apparent intracellular water signal remained constant within about $40 \mathrm{~s}$ after cardiac arrest and increased rapidly within a further $60 \mathrm{~s}$ to about $200 \%$ relative to the signal in the healthy brain.

Table 1

Mean apparent diffusion coefficient $D^{\text {app }}$ and its coefficient of variation $\sigma$, and relative fraction $p^{\text {app }}$ of the three peaks in the diffusogram of Fig. 5 as calculated by CONTIN from the rat brain CT data in the $x$ direction

Healthy rat brain Global ischemic rat brain

\begin{tabular}{|c|c|c|c|c|c|c|c|}
\hline$D^{\text {app }}\left(\mu \mathrm{m}^{2} / \mathrm{ms}\right)$ & $\sigma$ & $\Delta D^{\text {app a }}$ & $p_{2}^{\text {app }}(\%)$ & $D^{\text {app }}\left(\mu \mathrm{m}^{2} / \mathrm{ms}\right)$ & $\sigma$ & $\Delta D^{\text {app }}$ & $p_{2}^{\text {app }}(\%)$ \\
\hline $0.99 \pm 0.07$ & 0.62 & $0.5 \ldots 2.0$ & $95 \pm 2$ & $0.55 \pm 0.05$ & 0.71 & $0.3 \ldots 1.2$ & $92 \pm 1$ \\
\hline $0.08 \pm 0.01$ & 0.31 & $0.06 \ldots 0.11$ & $2.8 \pm 0.1$ & $0.045 \pm 0.006$ & 0.37 & $0.03 \ldots 0.07$ & $5.0 \pm 0.5$ \\
\hline
\end{tabular}

${ }^{\text {a }}$ The width $\Delta D^{\text {app }}$ is calculated from $\sigma$ and corresponds roughly to the FWHM of the peaks in the distribution. 
Table 2

Analysis of the multiexponential decay of the data in Fig. 4 by SplMod resulted in four components characterized by the apparent diffusion coefficient $D^{\text {app }}$ and its relative fraction $p^{\text {app a }}$

\begin{tabular}{lcll}
\hline Direction & $\begin{array}{l}D^{\text {app }}\left(\mu \mathrm{m}^{2} /\right. \\
\mathrm{ms})\end{array}$ & $\begin{array}{l}\text { Healthy rat } \\
\text { brain } p^{\text {app }}(\%)\end{array}$ & $\begin{array}{l}\text { Ischemic rat brain } \\
p^{\text {app }}(\%)\end{array}$ \\
\hline$x$ & $1.06 \pm 0.02$ & $62 \pm 1$ & $22 \pm 1$ \\
& $0.43 \pm 0.01$ & $32 \pm 1$ & $51 \pm 1$ \\
& $0.15 \pm 0.01$ & $4.2 \pm 0.4$ & $21 \pm 1$ \\
$y$ & $0.013 \pm 0.001$ & $2.6 \pm 0.1$ & $5.9 \pm 0.1$ \\
& $0.85 \pm 0.01$ & $67 \pm 1$ & $25 \pm 1$ \\
& $0.32 \pm 0.01$ & $24 \pm 1$ & $50 \pm 1$ \\
& $0.10 \pm 0.01$ & $5.9 \pm 0.4$ & $17 \pm 1$ \\
& $0.011 \pm 0.001$ & $3.5 \pm 0.2$ & $8.1 \pm 0.4$ \\
& $1.13 \pm 0.01$ & $70 \pm 1$ & $28 \pm 1$ \\
& $0.44 \pm 0.01$ & $25 \pm 1$ & $52 \pm 1$ \\
& $0.12 \pm 0.01$ & $3.7 \pm 0.2$ & $14 \pm 1$ \\
& $0.011 \pm 0.001$ & $1.9 \pm 0.1$ & $4.8 \pm 0.2$ \\
\hline
\end{tabular}

a Each pair of healthy and ischemic data was fitted simultaneously, whereby $D^{\text {app }}$ was only allowed one common (equal) value.

\subsection{Directional anisotropy}

The CT curves in Fig. 4 were also used to investigate the directional anisotropy of the water signal attenuation. ADCs, $D^{\text {app }}$, and the inherent apparent volume fractions, $p^{\text {app }}$, were calculated (Table 3 ) from the slope and intercept by log-linear regression in the range $b=$ $0-2 \mathrm{~ms} / \mu \mathrm{m}^{2}$ (attributed to index $1, \mathrm{ADC}$ ) and in the range $b=40-70 \mathrm{~ms} / \mu \mathrm{m}^{2}$ (attributed to index $2, D_{2}^{\text {app }}$ ). This procedure was performed for all three directions $x$, $y$, and $z$, as well as for the healthy and global ischemic rat brain data. A considerable difference in ADC in all directions could be observed, which was well beyond the statistical error. Although the absolute values of the ADC decreased during ischemia, the anisotropy ratio of the ADC in different directions was not altered and remained stable within $1 \mathrm{~h}$ after cardiac arrest within the statistical deviation.

However, at large $b$ values, no significant difference of $D_{2}^{\text {app }}$ in $x, y$, and $z$ was detectable within experimental error, whether in the healthy or the global ischemic rat brain data. However, the concomitant intercept, the apparent volume fraction $p_{2}^{\text {app }}$, showed directional anisotropy and its absolute value increased strongly during ischemia to about $250 \%$ relative to the healthy rat brain. In addition, the observed directional anisotropy of the ADC and $p_{2}^{\text {app }}$ depended on the position of the voxel in brain (data not shown); the overall results, however, and the amount of anisotropy of ADC and $p_{2}^{\text {app }}$ in contrast to $D_{2}^{\text {app }}$ were similar.

\subsection{Influences of diffusion time and $T_{2}$ relaxation}

The dependence of the water signal attenuation on the diffusion time was determined by $\mathrm{CT}$ experiments at $t_{\mathrm{D}}=57.7,117.7,177.7$, and $297.7 \mathrm{~ms}$ measured with identical $q$ range and plotted vs. $b$ and $q$ values (Fig. $7 \mathrm{~A}, \mathrm{~B})$. With increasing diffusion time, the signal at larger $b$ values decreased, whereas the $\mathrm{ADC}=0.70 \pm$ $0.03,0.76 \pm 0.03,0.78 \pm 0.05,0.77 \pm 0.06 \mu \mathrm{m}^{2} / \mathrm{ms}$, calculated in the range $b=0-2 \mathrm{~ms} / \mu \mathrm{m}^{2}$, slightly increased. From the plot vs. $q$ value, the apparent displacement was determined to be $r^{\text {app }}=5.4 \pm 0.1,4.7 \pm 0.3,4.0 \pm$ $0.5,4.8 \pm 0.2 \mu \mathrm{m}$ in the range $q=0.13-0.3 \mu \mathrm{m}^{-2}$. These values of $r^{\text {app }}$ around $5 \mu \mathrm{m}$ were connected to the apparent intracellular diffusion coefficient $D_{2}^{\mathrm{app}}=$ $0.085 \pm 0.004, \quad 0.032 \pm 0.004, \quad 0.015 \pm 0.004, \quad 0.013 \pm$ $0.001 \mu \mathrm{m}^{2} / \mathrm{ms}$ at large $q$ values, which decreased strongly with diffusion time. The overall constant apparent displacement $r$ app at diffusion times from about 60 to $300 \mathrm{~ms}$ pointed to a restricted diffusion characteristic of the mainly intracellular signal at $q>0.1 \mu \mathrm{m}^{-2}$. Additionally, from the signal decay with increasing diffusion time, a mean residence time of water was calculated to be $\tau^{\text {app }}=117 \pm 4 \mathrm{~ms}$ in the compartments detected at large $q$ values.

Potential compartmentation effects due to different relaxation times $T_{2}$ were investigated in the rat brain in vivo by a $\mathrm{CT}$ series at different echo times $\mathrm{TE}=20,50$, 80 , and $120 \mathrm{~ms}$, with identical diffusion weighting up to $b=40 \mathrm{~ms} / \mu \mathrm{m}^{2}\left(\delta=7 \mathrm{~ms}, t_{\mathrm{D}}=117.7 \mathrm{~ms}\right.$, data not shown). The signal decay was fitted at each $b$ value to possibly detect different $T_{2}$ at different diffusion weighting in rat brain tissue. However, $T_{2}=39 \pm 1 \mathrm{~ms}$, averaged over the total $b$ range, revealed only a small standard deviation, varied between a minimum and maximum of 36.7 and $41.0 \mathrm{~ms}$, and was thereby considered to be constant at all $b$ values.

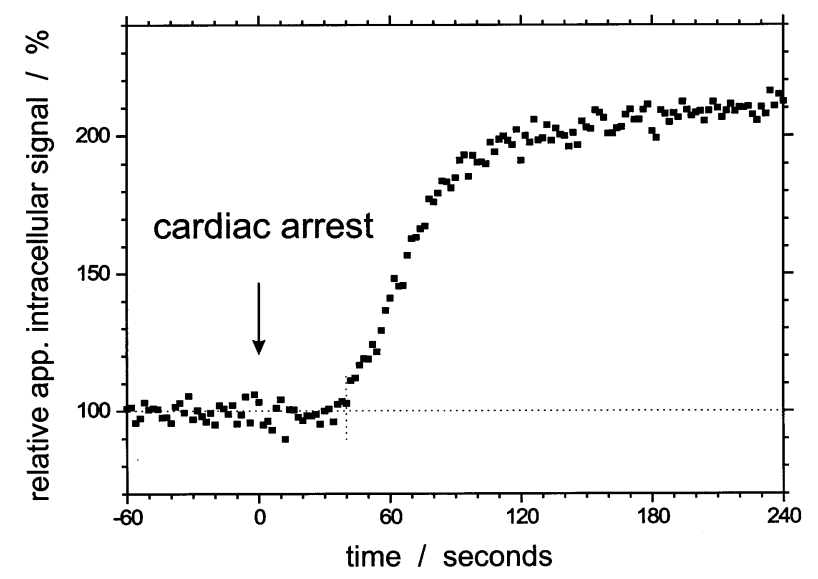

Fig. 6. Time-resolved single signal monitoring of the strong diffusionweighted water signal during the transition from healthy to the global ischemic rat brain in a $63 \mu \mathrm{l}$ voxel. The apparent intracellular water signal at $b=13.3 \mathrm{~ms} / \mu \mathrm{m}^{2}$ remained constant within $40 \mathrm{~s}$ after cardiac arrest $(t=0)$ and then increased rapidly within $60 \mathrm{~s}$. This indicated that the underlying mechanism of the ADC change during transient ischemia is a fast process, i.e. the shift from extracellular to intracellular water driven by an osmotic gradient. Experimental parameters: $G=300 \mathrm{mT} / \mathrm{m}, \delta=8 \mathrm{~ms}, t_{\mathrm{D}}=32 \mathrm{~ms}, \mathrm{TE}=22 \mathrm{~ms}, \mathrm{nt}=1, \mathrm{TR}=2 \mathrm{~s}$. 
Table 3

Apparent diffusion coefficient ADC and $D^{\text {app }}$ and apparent volume fraction $p^{\text {app }}$ and their standard deviation $\sigma$ calculated by log-linear regression from the data in Fig. $4^{\mathrm{a}}$

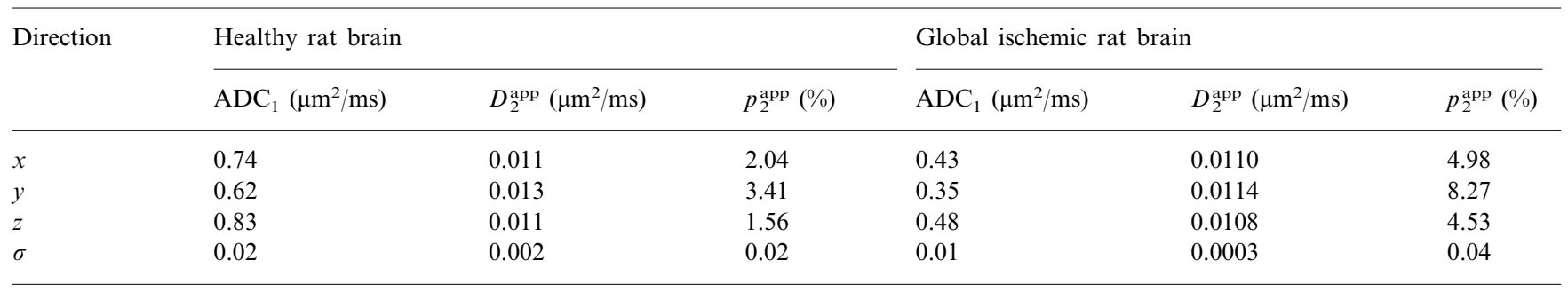

a The selected ranges for regression, $b=0-2$ and $40-70 \mu \mathrm{m}^{2} / \mathrm{ms}$, correspond to component (1) and (2), respectively.

\section{Discussion}

The feasibility of the in vivo detection of a very strongly diffusion-weighted water signal has been demonstrated in rat brain at $b=300 \mathrm{~ms} / \mu \mathrm{m}^{2}$. Due to the sensitivity of the pulsed-field-gradient sequence to any type of motion, this signal originates from water molecules that experience significant restriction in their movement, even within a time of about $400 \mathrm{~ms}$ of diffusion encoding. This is phenomenologically described by the apparent diffusion coefficient $D^{\text {app }}$, which is a factor 300 lower than that of freely diffusing water. The mean apparent displacement, calculated to be $5 \mu \mathrm{m}$, corresponds to the typical dimension of neuronal and glial cells in tissue, although one has to keep in mind that these values are ensemble averages over a spatial extension, which is three orders of magnitude larger than the cell dimensions and in this sense, a crude macroscopic average. In experiments on immobilized cells $[35,48,49]$, it has been shown for water and metabolites that a predominantly intracellular signal is detected at a diffusion weighting larger than approximately $b=5 \mathrm{~ms} / \mu \mathrm{m}^{2}$ due to the lowered $D^{\text {app }}$ when the molecules experience the cell membranes as restrictions and are reflected. In contrast, $D^{\text {app }}$ of the extracellular, free or hindered diffusing molecules in the surrounding perfusion medium or in the interstitial space in tissue is near the free diffusion constant and the inherent NMR signal decays fast at small $q$ values. At large $q$ values, the exchange of intracellular molecules can then be assessed from the signal decay with increasing diffusion time [18]. This model developed for cell data was extended to brain tissue by additionally including the extracellular hindered diffusion and its changes during ischemia, which is described by a tortuosity parameter $[5,50]$.

\subsection{Multiexponential analysis}

Our in vivo rat brain data with $b$ values up to 70 $\mathrm{ms} / \mu \mathrm{m}^{2}$ require multiple components using a discrete multiexponential analysis. This is consistent with observations in many different biological samples (see, for example, Refs.[9,24,51,52]), which were measured in an extended $b$ range and where multiexponential curves were fitted with two or three components. The multiexponential analysis in our study, resulting in at least four discrete components (DISCRETE, SplMod) or
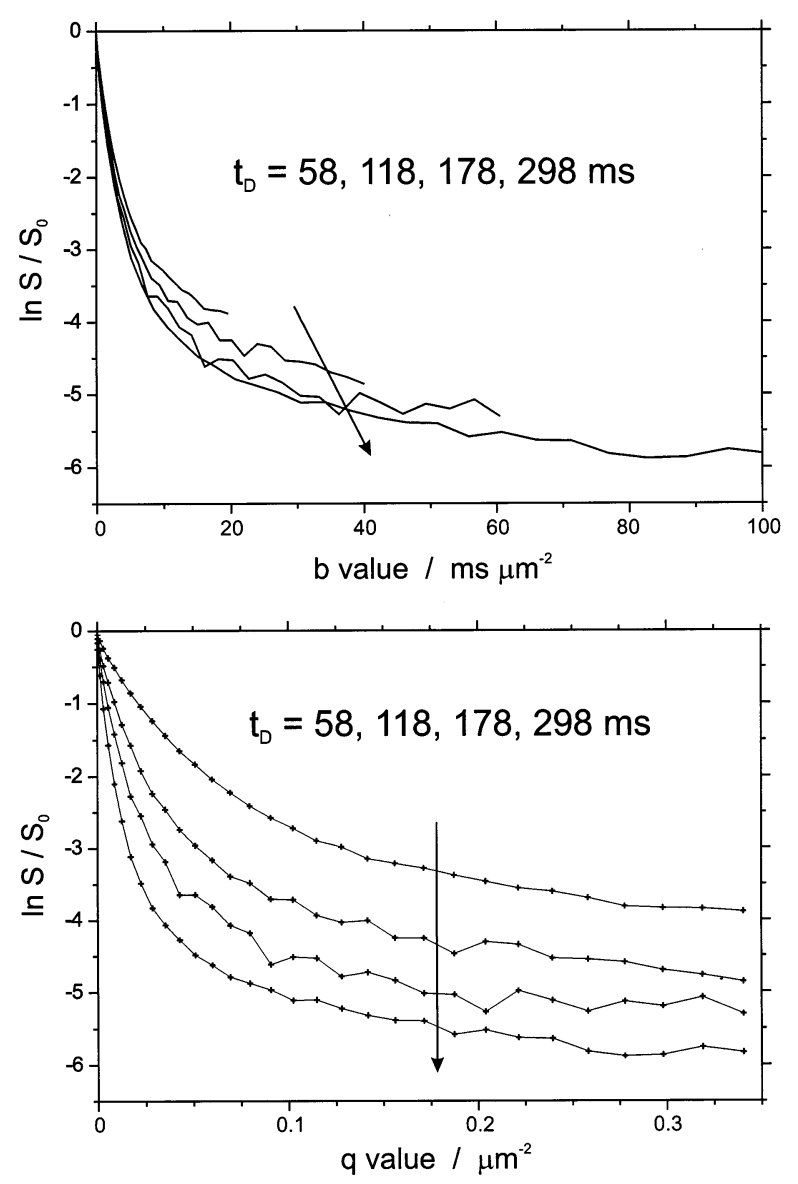

Fig. 7. Diffusion time dependence of the diffusion-weighted water signal in rat brain in vivo acquired with diffusion-weighted STEAM from a single $63 \mu \mathrm{l}$ voxel with $t_{\mathrm{D}}=57.7,117.7,177.7$, and $297.7 \mathrm{~ms}$. The constant slope in the plot vs. $q$ value indicated restricted diffusion of the mainly intracellular signal as detected at large $q$ values. The signal decrease with increasing diffusion time is due to exchange in the intracellular water compartments. The CT curves were normalized by $S(b=0)$ and recorded with $\delta=7 \mathrm{~ms}, \mathrm{TE}=20 \mathrm{~ms}, \mathrm{nt}=8$, $\mathrm{TR}=4 \mathrm{~s}$. 
a quasi-continuous diffusogram representing a sum of multiple exponentials (CONTIN), was able to describe the signal attenuation curves numerically, but the interpretation and assignment to physiological intracellular and extracellular compartments is not straightforward.

In this regard, the question arises whether a model of a discrete compartmentalization should still be considered valid and whether to assign the fitted decay coefficients to inherent diffusion coefficients $D^{\text {app }}$, which are to describe the motion characteristic in two, three, four or more compartments. In contrast, the water diffusion attenuation is commonly measured in a small $b$ range and analyzed by a monoexponentially fitted ADC, which provides a sensitive NMR imaging contrast parameter to detect tissue swelling during ischemia.

\subsection{Multicompartmentalization and morphology}

Additionally, we used a previously demonstrated two-compartment model [18] with intracellular restricted and extracellular hindered diffusion, including exchange for simulation (not shown). The signal attenuation $S=S\left(q, t_{\mathrm{D}}, D_{1}, D_{2}, p_{2}, \tau_{2}, \lambda, a, \sigma_{a}\right)$ was calculated as a function of the experimental parameters $q$ value and diffusion time $t_{\mathrm{D}}$, on the physiological parameters $D_{1}, D_{2}, p_{2}, \tau_{2}$ (extracellular and intracellular diffusion constant, intracellular volume fraction and exchange time), and on the morphological parameters $\lambda$ (tortuosity factor) and $a$ (box length). The model data were only consistent with the experimental diffusion attenuation curves when a distribution of box lengths characterized by a Gaussian distribution width $\sigma_{a}$ was included.

The major results were that: (1) the diffusion attenuation curves were nearly fully insensitive to variations of the intrinsic intracellular diffusion constant $D_{2}$ in the range $2.0-0.2 \mu \mathrm{m}^{2} / \mathrm{ms}$; (2) the intracellular exchange time $\tau_{2}$ also revealed minimal influence on the ADC at small $q$ values, but affected the volume fraction at large $q$ values depending on the diffusion time; (3) all simulated curves were very sensitive to changes of the volume fraction $p_{2}$ : the ADC at small $q$ values decreased and $p_{2}^{\text {app }}$ increased with increasing $p_{2}$; (4) the absolute value of the ADC at small $q$ values was sensitive to the extracellular diffusion constant $D_{1}$ and the tortuosity coefficient $\lambda$ and was sensitive to the size and the distribution width of the restricting boundaries, i.e. the box length $a$ and $\sigma_{a}$ in the model; (5) an analysis by CONTIN revealed also three and more distribution peaks at 1 , 0.1 , and $0.01 \mu \mathrm{m}^{2} / \mathrm{ms}$, although only two main compartments were modeled. One may conclude that the morphology and cell size distribution determines mainly the ' $q$ space diffusion diffraction' $[28,53]$ pat- terns of the attenuation curves and that the ADC reflects changes in the restricting boundaries, i.e. mainly the relative volume fractions and the extracellular tortuosity.

In this sense, the experimental data show apparent multicompartmental properties and a more complicated model has to be used when involving morphological features. These imply $q$ space distribution functions, which reflect the shape of the restricting boundaries and which are, in general, non-exponential functions, e.g. as described by the parameters pore length and pore connectivity in the theory of porous media $[30,32,33,54]$. Additionally, the size of these morphological objects is altered in biological samples due to growth processes or different functionality, which superposes an arbitrary, complicated size distribution function. A further extension of the presented modeling may include the permeability of the boundaries rather than the intracellular exchange time, similar to the random walk simulations in Ref. [16]. With a distribution of cell diameters at constant wall permeability, the exchange times are supposed to change accordingly. In this context, a directionally anisotropic exchange time due to morphological anisotropy, e.g. in axons and cell bodies, can be considered.

The directional anisotropy of the diffusion attenuation is an example of a macroscopic coherent morphology, which has been represented by a NMR detectable diffusion tensor, which is less a tensor of inherently different diffusion constants than a tensor of anisotropic morphology and restriction dimensions. To incorporate these properties into the modeling, a superposition of signals arising from multiple compartments with different cell dimensions has been used and as a simple approximation a Gaussian distribution of box lengths has been assumed $[12,18]$.

\subsection{Physiological interpretation}

The diffusograms presented here reveal that a major volume fraction of $D^{\text {app }}$ of about $90-95 \%$ is subsumed under one broad distribution peak. Two minor fractions with $D^{\text {app }}$ one and two orders of magnitude lowered are significantly separated. The interpretation as distinct physical environments is not excluded, but also not straightforward or evident from the diffusogram or the discrete analysis. The comparison of the DISCRETE, SplMod, and CONTIN analysis indicates that the major peak in the diffusogram may be separable into two components, the first of which decreases in volume fraction during ischemia, while the second increases. Although a direct quantitative relationship remains to be established, it is conceivable that the contribution of extracellular and intracellular water to these two components is significantly different, being 
consistent with a shift from extra- to intracellular water during ischemia.

Recent investigation of diffusional anisotropy of $T_{2}$ components in bovine optic nerve at 1.5 Tesla [13] resulted in two different intracellular compartments: one with short $T_{2}=13.5 \mathrm{~ms}$ and minor anisotropy associated with water within the myelin sheath, the second with long $T_{2}=134 \mathrm{~ms}$ and major anisotropy to intra-axonal water. Triexponential water diffusion attenuation in excised brain tissue was demonstrated in a wide range of diffusion times attributed to two compartments with restricted diffusion ( $r$ app approximately 2 and $6 \mu \mathrm{m}$ ), and one with almost free or hindered diffusion. Also, three different water populations with $D^{\text {app }}=1.2,0.35$, and approximately $0.03 \mu \mathrm{m}^{2} / \mathrm{ms}$ have been found at $25^{\circ} \mathrm{C}$ using deuterium single- and doublequantum filtering $[24,55,56]$.

To focus on the slowly decaying components, the time-resolved intracellular signal at $b=13.3 \mu \mathrm{m}^{2} / \mathrm{ms}$ was acquired during ischemia to monitor changes in the apparent intracellular volume fraction and thereby the tissue swelling, which revealed three stages: (1) during the first $40 \mathrm{~s}$ after cardiac arrest, remaining ATP and glucose reservoirs and protection mechanisms of the brain, i.e. energy storage phosphocreatine and glycogen, kept at least the intracellular volume fraction constant; (2) within one further minute there was a fast signal change attributed to swelling due to a breakdown of the tissue energy status and a shift from extra- to intracellular water caused by osmotic gradients; (3) the intracellular signal became again nearly constant and increased slightly within a long time scale of hours due to tissue degeneration (data not shown). The detection of the monitored intracellular signal and its changes can be directly correlated with the ADC changes (calculated usually in a $b$ range up to $1-2 \mathrm{~ms} / \mu \mathrm{m}^{2}$ ) because $S(b=0)$ did not change significantly (data not shown). This points to the fact that also the ADC follows the same time course and is mainly influenced by an energy dependent mechanism and by a fast shift of water between compartments, which is correlated with changes in the relative compartmental volume fractions. A further contribution to the ADC changes during ischemia is the increase of the tortuosity factor as a consequence of the reduced extracellular space $[6,12]$. Similar fast changes of the ADC were observed during spreading cortical depressions in rat brain induced by potassium chloride (see, for example, Ref. [57]), which are also correlated with transient changes in local volume fractions.

\subsection{Anisotropy, diffusion time and $T_{2}$ relaxation}

Anisotropic behavior depending on the gradient direction was found for the ADC at small $b$ values (Table $3)$. However, $D_{2}^{\text {app }}$ at large $b$ values was isotropic in contrast to the inherent volume fraction $p_{2}^{\text {app }}$, which was highly anisotropic. Compared with the behavior of $D^{\text {app }}$ and the volume fractions $p^{\text {app }}$ during ischemia (Table 2), it seemed that the volume fractions of the multiple compartments in tissue and its changes determine the anisotropy of the ADC as well as the changes of the ADC during ischemia. The very small absolute value of $D_{2}^{\text {app }}$ hinted to the influence of restrictions to these compartments. Together with the diffusion time dependent measurements, this additionally confirms the concept of 'restricted diffusion at permeable boundaries' in analogy to Ref. [12]. Compared with the examined $t_{\mathrm{D}}$ range $17-63 \mathrm{~ms}$ in Ref. [12], our $t_{\mathrm{D}}$-dependent data showed an additional signal decrease in the plot vs. $b$ value at the larger diffusion times $t_{\mathrm{D}}=58-$ $298 \mathrm{~ms}$ and a significantly larger mean apparent exchange time.

It was shown previously that different relaxation times $T_{1}$ or $T_{2}$ and different diffusion properties could be used to characterize and separate compartments $[58,59]$. In our echo time dependent experiments in rat brain in vivo, no alterations in $T_{2}=39 \pm 1 \mathrm{~ms}$ at different diffusion weighting could be detected, from which can be concluded that $T_{2}$ of the major extracellular compartment in rat brain is not significantly higher than intracellular $T_{2}$. The previously observed intracellular water component with very large $T_{2}$ in excised rat brain [25] could not be confirmed by this experiment in vivo. Components with short $T_{2}$, e.g. $11 \mathrm{~ms}$ in Ref. [13], were principally not detectable by our experiment due to the minimum echo time of $20 \mathrm{~ms}$.

\section{Conclusions}

Multiparameter analysis separating $q$ value, diffusion time, and directional anisotropy under healthy and global ischemic conditions was performed at very large diffusion weighting in the rat brain in vivo with high sensitivity. The multiexponential analysis revealed that a representation as a continuous distribution of diffusion coefficients, i.e. a diffusogram, is more reasonable than a discrete multiexponential model with at least four components. Considering the complicating effects of morphology and cell size distributions in tissue, a simple assignment of multiexponential components and their interpretation in terms of number of compartments and their intrinsic diffusion constants, etc., is not straightforward. Highly restricted components separated at large $q$ values showed only a change in volume fraction during global ischemia, whereas the apparent diffusion coefficient was not altered. The changes of the whole attenuation curves in ischemic rats could be fully described by changes in the relative volume fractions only, without changes in the apparent diffusion coefficients. In addition, the detected anisotropy of the ADC 
at small $q$ values was not observed for the apparent diffusion coefficient at large $q$ values, but for the adjacent volume fractions. This was consistent with an analytical model combining hindered and restricted diffusion, and exchange, in which the relative volume fractions and the tortuosity factor showed the most relevant effect on the ADC and reflect the changes in the restricting boundaries during ischemia. In contrast, changes of the intrinsic intracellular diffusion constant and the exchange time revealed a minor effect on the ADC. The demonstrated multimodal influences in vivo and ex vivo on the ADC measured over a wide range of $b$ values may lead to a further understanding of the changes in tissue properties and its assessment by NMR in normal and pathological states.

\section{Acknowledgements}

The authors thank Prof. Kamil Ugurbil and Prof. Michael Garwood, Minneapolis for generous support, and Prof. Dieter Leibfritz, Bremen for helpful discussion. Supported by NIH grants R01 CA64338 and P41 RR08079, and the W.M. Keck Foundation.

\section{References}

[1] Stejskal EO, Tanner JE. Spin diffusion measurements: spin echoes in the presence of a time-dependent field gradient. J Chem Phys 1965;42:288-92.

[2] Tanner JE, Stejskal EO. Restricted self-diffusion of protons in colloidal systems by the pulsed-gradient, spin-echo method. J Chem Phys 1968:49:1768-77.

[3] Moseley ME, Butts K, Yenari MA, Marks M, de Crespigny A. Clinical aspects of DWI. NMR Biomed 1995;8:387-96.

[4] Anderson AW, Zhong J, Petroff OA, Szafer A, Ransom BR, Prichard JW, Gore JC. Effects of osmotically driven cell volume changes on diffusion-weighted imaging of the rat optic nerve. Magn Reson Med 1996;35:162-7.

[5] Nicholson C, Phillips JM. Ion diffusion modified by tortuosity and volume fraction in the extracellular microenvironment of the rat cerebellum. J Physiol (Lond) 1981;321:225-57.

[6] van der Toorn A, Sykova E, Dijkhuizen RM, Vorisek I, Vargova L, Skobisova E, van Lookeren C, Reese T, Nicolay K. Dynamic changes in water ADC, energy metabolism, extracellular space volume, and tortuosity in neonatal rat brain during global ischemia. Magn Reson Med 1996;36:52-60.

[7] Nicholson C, Sykova E. Extracellular space structure revealed by diffusion analysis. Trends Neurosci 1998;21:207-15.

[8] Helmer KG, Dardzinski BJ, Sotak CH. The application of porous-media theory to the investigation of time- dependent diffusion in in vivo systems. NMR Biomed 1995;8:297-306.

[9] Stanisz GJ, Szafer A, Wright GA, Henkelman RM. An analytical model of restricted diffusion in bovine optic nerve. Magn Reson Med 1997;37:103-11.

[10] Helpern JA, Ordidge RJ, Knight RA. The effect of cell membrane water permeability on the apparent diffusion coefficient of water. Proceedings of the ISMRM 11th Annual Meeting, Berlin, 1992, p. 1201.

[11] Waldeck AR, Nouri-Sorkhabi MH, Sullivan DR, Kuchel PW.
Effects of cholesterol on transmembrane water diffusion in human erythrocytes measured using pulsed field gradient NMR. Biophys Chem 1995;55:197-208.

[12] Pfeuffer J, Dreher W, Sykova E, Leibfritz D. Water signal attenuation in diffusion-weighted ${ }^{1} \mathrm{H}$ NMR experiments during cerebral ischemia: influence of intracellular restrictions, extracellular tortuosity, and exchange. Magn Reson Imaging 1998;16:1023-32.

[13] Stanisz GJ, Henkelman RM. Diffusional anisotropy of $T_{2}$ components in bovine optic nerve. Magn Reson Med 1998;40:40510.

[14] Duong TQ, Ackerman JJ, Ying HS, Neil JJ. Evaluation of extraand intracellular apparent diffusion in normal and globally ischemic rat brain via ${ }^{19} \mathrm{~F}$ NMR. Magn Reson Med 1998;40:113.

[15] Latour LL, Svoboda K, Mitra PP, Sotak CH. Time-dependent diffusion of water in a biological model system. Proc Natl Acad Sci USA 1994;91:1229-33.

[16] Szafer A, Zhong J, Gore JC. Theoretical model for water diffusion in tissues. Magn Reson Med 1995;33:697-712.

[17] Pfeuffer J, Norris DG, Niendorf T, Leibfritz D. Monte Carlo modelling of pulsed gradient spin echo experiments in ischemic brain tissue. Proceedings of the ISMRM 3rd Scientific Meeting, Nice, 1995, p. 1382.

[18] Pfeuffer J, Flögel U, Dreher W, Leibfritz D. Restricted diffusion and exchange of intracellular water: theoretical modelling and diffusion time dependence of ${ }^{1} \mathrm{H}$ NMR measurements on perfused glial cells. NMR Biomed 1998;11:19-31.

[19] Price WS, Barzykin AV, Hayamizu K, Tachiya M. A model for diffusive transport through a spherical interface probed by pulsed-field gradient NMR. Biophys J 1998;74:2259-71.

[20] Horsfield MA, Barker GJ, McDonald WI. Self-diffusion in CNS tissue by volume-selective proton NMR. Magn Reson Med 1994;31:637-44.

[21] Basser PJ, Pierpaoli C. Microstructural and physiological features of tissues elucidated by quantitative-diffusion-tensor MRI. J Magn Reson B 1996;111:209-19.

[22] Pierpaoli C, Basser PJ. Toward a quantitative assessment of diffusion anisotropy. Magn Reson Med 1996;36:893-906 [published erratum appears in Magn Reson Med 1997;37(6):972].

[23] Niendorf T, Norris DG, Leibfritz D. Detection of apparent restricted diffusion in healthy rat brain at short diffusion times. Magn Reson Med 1994;32:672-7.

[24] Assaf Y, Cohen Y. Non-mono-exponential attenuation of water and $\mathrm{N}$-acetyl aspartate signals due to diffusion in brain tissue. $\mathrm{J}$ Magn Reson 1998;131:69-85.

[25] Assaf Y, Cohen Y. Diffusion MRS and MRI of fibers in bovine optic nerve and in rat brain in vivo. Proceedings of the ISMRM 6th Scientific Meeting, Sydney, 1998, p. 1263.

[26] King MD, Houseman J, Roussel SA, van Bruggen N, Williams SR, Gadian DG. q-Space imaging of the brain. Magn Reson Med 1994;32:707-13.

[27] King MD, Houseman J, Gadian DG, Connelly A. Localized q-space imaging of the mouse brain. Magn Reson Med 1997;38:930-7.

[28] Kuchel PW, Coy A, Stilbs P. NMR 'diffusion-diffraction' of water revealing alignment of erythrocytes in a magnetic field and their dimensions and membrane transport characteristics. Magn Reson Med 1997;37:637-43.

[29] Niendorf T, Dijkhuizen RM, Norris DG, van Lookeren C, Nicolay K. Biexponential diffusion attenuation in various states of brain tissue: implications for diffusion-weighted imaging. Magn Reson Med 1996;36:847-57.

[30] Mitra PP, Sen PN. Effects of microgeometry and surface relaxation on NMR pulsed-field-gradient experiments: simple pore geometries. Phys Rev B 1992;45:143-56. 
[31] Mitra PP, Sen PN, Schwartz LM, Doussal PL. Diffusion propagator as a probe of the structure of porous media. Phys Rev Lett 1992;68:3555-8.

[32] Callaghan PT, Coy A, MacGowan D, Packer KJ, Zelaya FO. Diffraction-like effects in NMR diffusion studies of fluids in porous solids. Nature 1991;351:467-9.

[33] Callaghan PT. NMR imaging, NMR diffraction and applications of pulsed gradient spin echoes in porous media. Magn Reson Imaging 1996;14:701-9.

[34] Pfeuffer J. Beschränkte Diffusion und Austausch von Wasser in Zellkulturen und im Gehirn: Theoretische Modelle und ${ }^{1} \mathrm{H}$ NMR-Messungen. Dissertation, Universität Bremen Shaker Verlag Aachen, 1996.

[35] Pfeuffer J, Flögel U, Leibfritz D. Monitoring of cell volume and water exchange time in perfused cells by diffusion-weighted ${ }^{1} \mathrm{H}$ NMR spectroscopy. NMR Biomed 1998;11:11-8.

[36] Pfeuffer J, Flögel U, Leibfritz D. Influences of diffusion time and osmotic stress on intracellular metabolite signals in perfused glial cells as detected by diffusion-weighted ${ }^{1} \mathrm{H}$ NMR Spectroscopy. Proceedings of the ISMRM 6th Scientific Meeting, Sydney, 1998, p. 533.

[37] Tkac I, Starcuk Z, Choi I-Y, Gruetter R. In vivo ${ }^{1} \mathrm{H}$ NMR spectroscopy of rat brain at $1 \mathrm{~ms}$ echo time. Magn Reson Med 1999;41:649-56.

[38] Merboldt KD, Hanicke W, Frahm J. Diffusion imaging using stimulated echoes. Magn Reson Med 1991;19:233-9.

[39] Terpstra M, Andersen PM, Gruetter R. Localized eddy current compensation using quantitative field mapping. J Magn Reson 1998;131:139-43.

[40] Gruetter R. Automatic, localized in vivo adjustment of all firstand second-order shim coils. Magn Reson Med 1993;29:804-11.

[41] Provencher SW. An eigenfunction expansion method for the analysis of exponential decay curves. J Chem Phys 1976;64:2772-7.

[42] Provencher SW, Vogel RH. Information loss with transform methods in system identification: a new set of transforms with high information content. Math Biosci 1980;50:251-62.

[43] Provencher SW, Vogel RH. Regularization techniques for inverse problems in molecular biology. In: Deuflhard P, Hairer E, editors. Numerical treatment of inverse problems in differential and integral equations. Boston: Birkhäuser, 1983:304-19.

[44] Provencher SW. A constrained regularization method for inverting data represented by linear algebraic or integral equations. Comput Phys Commun 1982;27:213-27.

[45] Provencher SW. CONTIN: a general purpose constrained regularization program for inverting noisy linear algebraic and integral equations. Comput Phys Commun 1982;27:229-42.

[46] Labadie C, Lee JH, Vetek G, Springer CS. Relaxographic imaging. J Magn Reson B 1994;105:99-112.
[47] Weingartner H. Self diffusion in liquid water. a reassessment. Z Phys Chem 1982;132:129-49.

[48] van Zijl PC, Moonen CT, Faustino P, Pekar J, Kaplan O, Cohen JS. Complete separation of intracellular and extracellular information in NMR spectra of perfused cells by diffusionweighted spectroscopy. Proc Natl Acad Sci USA 1991;88:322832.

[49] Flögel U, Niendorf T, Serkowa N, Brand A, Henke J, Leibfritz D. Changes in organic solutes, volume, energy state, and metabolism associated with osmotic stress in a glial cell line: a multinuclear NMR study. Neurochem Res 1995;20:793-802.

[50] Lehmenkuhler A, Sykova E, Svoboda J, Zilles K, Nicholson C. Extracellular space parameters in the rat neocortex and subcortical white matter during postnatal development determined by diffusion analysis. Neuroscience 1993;55:339-51.

[51] van der Veen JW, Van Gelderen P, Creyghton JH, Bovee WM. Diffusion in red blood cell suspensions: separation of the intracellular and extracellular NMR sodium signal. Magn Reson Med 1993;29:571-4.

[52] Pilatus U, Shim H, Artemov D, Davis D, van Zijl PC, Glickson JD. Intracellular volume and apparent diffusion constants of perfused cancer cell cultures, as measured by NMR. Magn Reson Med 1997;37:825-32.

[53] Cory DG, Garroway AN. Measurement of translational displacement probabilities by NMR: an indicator of compartmentation. Magn Reson Med 1990;14:435-44.

[54] Sen PN, Schwartz LM, Mitra PP, Halperin BI. Surface relaxation and the long-time diffusion coefficient in porous media: periodic geometries. Phys Rev B 1994;49:215-25.

[55] Assaf Y, Cohen Y. Detection of different water populations in brain tissue using $2 \mathrm{H}$ single- and double-quantum-filtered diffusion NMR spectroscopy. J Magn Reson B 1996;112:151-9.

[56] Assaf Y, Navon G, Cohen Y. In vivo observation of anisotropic motion of brain water using $2 \mathrm{H}$ double quantum filtered NMR spectroscopy. Magn Reson Med 1997;37:197-203.

[57] Latour LL, Hasegawa Y, Formato JE, Fisher M, Sotak $\mathrm{CH}$. Spreading waves of decreased diffusion coefficient after cortical stimulation in the rat brain. Magn Reson Med 1994:32:189-98.

[58] Pfeuffer J, Bröer S, Bröer A, Lechte M, Flögel U, Leibfritz D. Expression of aquaporins in Xenopus laevis oocytes and glial cells as detected by diffusion-weighted ${ }^{1} \mathrm{H}$ NMR spectroscopy and photometric swelling assay. Biochim Biophys Acta 1998;1448:27-36.

[59] Han SS, Vetek G, Springer CS, Sotak CH. Apparent diffusion coefficient of intra- and extracellular water in yeast suspension measured by combined diffusion and relaxography. Proceedings of the ISMRM 6th Scientific Meeting, Sydney, 1998, p. 535 . 